\title{
COMPARATIVE STUDY OF SOLAR OPTICAL FIBRE AND ARTIFICIAL LIGHT FOR DAYLIGHTING IN TUNNEL THRESHOLD ZONE
}

\author{
Canan Perdahci ${ }^{1}$ and Yahya Sogodok ${ }^{2}$
}

\author{
Kocaeli University, Department of Electrical Engineering, Kocaeli, Turkey

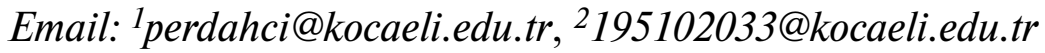

\begin{abstract}
In recent years, there has been a considerable increase in traffic accidents in the tunnel region, especially around the threshold and exit zones, due to the inappropriate lighting system of these zones; leading to the loss of humans properties and lives. Therefore, lighting designers strive to improve the illumination issues of the threshold and exit zones which bring about "black hole" and "white hole" effects, respectively. Several solutions are proposed: solar optical fibre lighting system as a natural lighting system and LED, fluorescent lighting system as the artificial lighting system. This paper aims to compare the natural and artificial lighting system to show which lighting system is better and the best solution to solve these issues. The previous study in the Huashuyan tunnel in China, where natural light, solar optical fibre was used to illuminate the threshold zone, is simulated in LITSTAR4D software using a customized HCL-TN LED luminaire of LITPA Company. The simulation result is compared with the previous result obtained when the solar optical fibre is used. The simulation outcome gave a satisfying result with an average luminance of $91.15 \mathrm{~cd} / \mathrm{m}^{2}$, which is higher than the average luminance required by the threshold zone. However, it is less when compared to the average luminance of $181.31 \mathrm{~cd} / \mathrm{m}^{2}$ produced by the solar optical fibre system. The overall uniformity of 0.42 is above the requirements in the CIE88:2004 standards.
\end{abstract}

Keywords: solar optic fibre, LED, threshold, lighting system, LITSTAR4D

\section{INTRODUCTION}

Daylighting for tunnel lighting is critical, which is too sensitive and needs careful study while designing.
The daylighting has a significant impact on human vision at the tunnel entrance and exit zones [1]. A driver leaving from a brighter environment to the tunnel witness's access zone or threshold zone what we call "black hole phenomena"; that is, the driver sees the tunnel as a black hole. At this point, the driver is in a critical situation; a slight mistake made will cause a fatal accident. This is why it is required to design the threshold zone lighting system to mimic the exterior light or the daylight so that the driver's eyes can adapt fast to the sudden change in illumination level. Therefore, many illumination methods are applied to mitigate this problem. For example, some countries like China adopted a natural form of lighting by using solar optical fibre for daylighting in the threshold zone [2], designed solar optical fibre to improve the illumination of the threshold zone during the day. This case study was conducted on the Huashuyan Tunnel of G109 Highway. They conducted both experimental and simulation to verify the correctness of the study. The luminance of the threshold region was varying with respect to the sunlight variation outside the tunnel. According to the calculation, the luminance requirement was approximately $83.47 \mathrm{~lx}$, but simulation showed that the output luminance was $181.31 \mathrm{~cd} / \mathrm{m}^{2}$, above the required value. The findings showed how efficient the proposed new lighting system was.

Similarly, in many tunnels today, better luminaires such as the LED luminaires are used due to their excellent optical performances, energy-saving capability, and reduce greenhouse effects. In [3], a study is done to determine the reaction time under different luminaires like high sodium, metal halides, and LED luminaires. LED luminaires in the tunnels provide the shortest reaction time compared to high-pressure sodium and metal halide luminaires. In this study, customized HCL-TN LED luminaires are used in the simulation software to calculate 
the average luminance requirement of the threshold zone. The result is compared with the previous result when a solar optical fibre lighting system was used [2].

\section{COMPONENTS AND METHOD OF DAYLIGHTING USING OPTICAL FIBRE}

Optical fibre is used as a transmission medium in the studied system of the Huashuyan Tunnel[2]. The captured light was directed into the tunnel through the fibre optic using the total internal reflection of light. The daylighting consisted of the components described below.

\subsection{Solar Collector}

There is much technology of solar collector proposed in much research among which are the next, presented below.

\subsubsection{Parans solar collector}

Paran is a Swedish Company specialising in the production of lighting equipment both for buildings and road lighting. The company also developed lighting devices for tunnel lighting in recent years and is currently designing tunnel lighting in the entrance zone in the Netherland in the region of the Rijnlands tunnel [4]. The Parans solar collector is shown in Fig. 1. The sun collector is equipped with a sun-tracking system and is connected to a computer to track the suns movement during the whole day to collect the maximum light possible to illuminate the tunnel. Furthermore, the sun collector is connected to the fibre optic, which receives the light from the concentrator and directs the light to the diffuser. The system's stepper motor used as a tracking system allows the collector to rotate horizontally 360 degrees on its axis and \pm 90 degrees.

\subsubsection{Himawari solar collector system}

The word Himawari is a Japanese word of 'Sunflower'. As the Sunflower faces the sun, hence the name was invented by Dr. Keo Mori, a Japanese Professor. The system consists of a lens used for focusing and a centralized optical sensor for tracking the sun position during the day; thus, the system can sufficiently provide the space in question enough light for illumination[5]. The system is shown in Fig. 2.

\subsection{Light Transmission System}

The fibre optic is used as a transmission system for light from the collector to the interior space. The system is based on the working principles of fibre optic. Here, the light travels in a zigzag fashion in the fibre optic, and the light bends at a particular angle known as the critical angle of usually 40 degrees. This zigzag movement of light in the fibre optic is known as the total internal reflection. Three major materials are used for fibre optic manufacturing: Quartz, the most efficient and most expensive material and lower attenuation constant $(0.2 \mathrm{~dB} / \mathrm{Km})$, the Plastic fibre optic also known as Polymethylmethacrylate (PMMA); the cheapest but with high losses and less efficient, and the optical fibre with a core made of highly purified liquids with a relatively higher attenuation constant of $2 \mathrm{~dB} / \mathrm{Km}$.
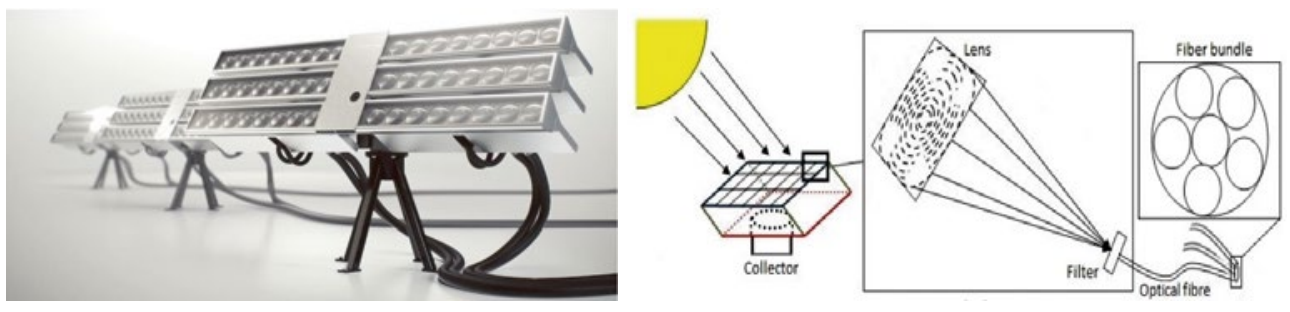

Fig. 1. Parans solar collector
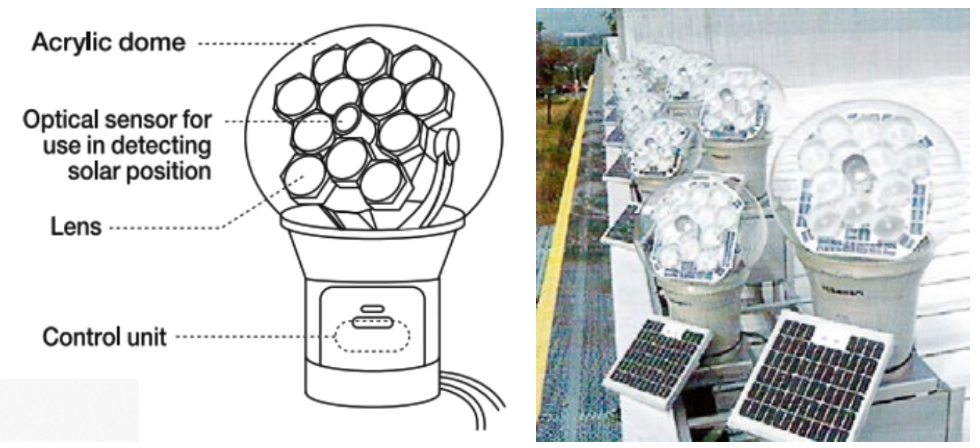

Fig. 2. Himawari solar collector 

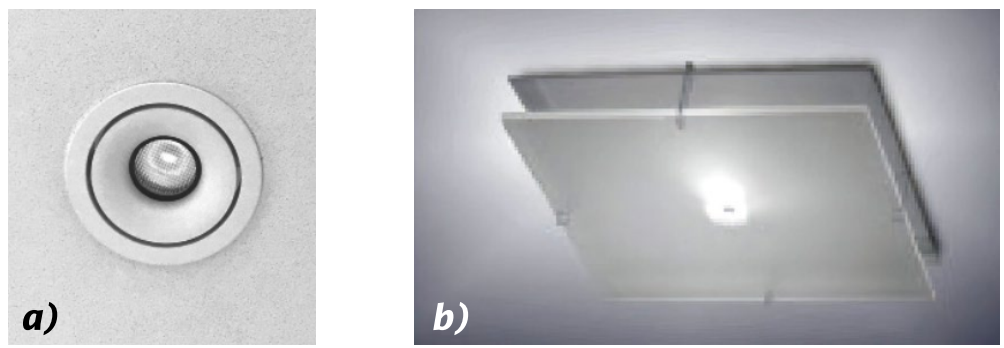

Fig. 3. Parans light diffuser, a) pure point light, b) hybrid point light
Plastic fibre optic is one of the most suitable for daylight; however, it has heating due to infrared radiation of the sunlight, which will break down the optical fibre at a certain point. However, the system which uses this transmission medium is equipped with a proper filter to filter out the IR and UV light [6].

\section{LIGHT DIFFUSER}

When light is transmitted to the tunnel's interior, a diffuser in the form of a luminaire is required to spreads the light and illuminates the surrounding area. The material of these luminaires should be of high quality to spread the light and surface hardness to last for several years to account for maintenance cost in the road tunnel. The diffuser materials can be: polycarbonate (PC), polystyrene (PS) and acrylic PMMA [7]. These materials have high optical parameters, which reduce transmission losses. The paran luminaires are shown in Fig. 3.

The light diffuser or luminaire can be categorized into two types: the hybrid luminaires, as shown in Fig. $3(b)$ and Fig. $3(a)$, which is passive. The hybrid is an efficient type of luminaire as it is used with electrical lighting, which helps compensate for the shortage of daylighting during the cloudy sky. This luminaire contains a sensor and a controller, which sense the availability of solar light so that the electrical light is switched on or off to avoid any visual discomfort.

In contrast, the passive luminaire is used only on a sunny day. Passive luminaire is only effective during a sunny day, and sufficient solar light is available.[6].

\section{SOLAR OPTICAL FIBRE LIGHTING SYSTEM DESIGN}

The solar optical lighting system is an efficient method of daylighting in the threshold zone of a tunnel. This provides suitable energy required by this zone and also contributes to energy saving during the day. Here, the solar collector direct light into the optical fibre cable. The optical fibre will transmit the light into the threshold zone by the principle of total internal reflection. Then, the light is spread to the working place by a diffuser at the end of the optical fibre irradiators. This study was done by [2] in the Huashuyan tunnel in China. The system was much efficient and provided the threshold zone with the required average luminance equal to $181.31 \mathrm{~cd} / \mathrm{m}^{2}$, which is higher than the average demand luminance equal to $83.47 \mathrm{~cd} / \mathrm{m}^{2}$.

The threshold zone was aligned by five pipes containing each fibre optic irradiator, which carries light from the exterior to the tunnel's interior, with the first pipe place $10 \mathrm{~m}$ from the entrance of the threshold zone. The interspacing between the endings of one pipe to the other is $5 \mathrm{~m}$. Then the luminance of the interior measured and the average luminance was $181.31 \mathrm{~cd} / \mathrm{m}^{2}$, which is an excellent value for the zone.

\section{DESIGN OF DAYLIGHTING OF THE THRESHOLD ZONE BY LED LUMINAIRE}

The LED luminaires are the most used type of luminaires today in the tunnel for daylighting due to their excellent photometric performance and their economic aspect for energy consumptions. The LED luminaire has a good colour rendering index, correlated colour temperature, luminance, and illuminance. These properties make it the best choice nowadays in both interior and exterior lighting design. Therefore, in this paper, the design of the threshold zone is done by using a customized LED luminaire by the LITPA lighting company [8]. The characteristics of the luminaire are given in Table 1.

\section{METHOD}

\subsection{Calculation of the Threshold Zone Parameters of the Tunnel}

To design the threshold zone in LITSTAR4D, we need some parameters such as the average luminance inside the tunnel, the luminance of the surrounding, stopping distance, clearance height, longitudinal slope, and the longitudinal speed of the tunnel. These parameters are given in [2] and are shown in Table 2. 
Table 1. Counteract-Beam Luminaire and Its Parameters

\begin{tabular}{|c|c|c|}
\hline \multirow{2}{*}{} & Code & HCL/TN200 \\
\cline { 2 - 3 } & LED & Power LED \\
\cline { 2 - 3 } & System power & $200 \mathrm{~W}$ \\
\cline { 2 - 3 } & Light flux & $26.120 \mathrm{~lm}$ \\
\cline { 2 - 3 } & Colour temperature & $3000 \mathrm{~K}-4000 \mathrm{~K}-5000 \mathrm{~K}$ \\
\cline { 2 - 3 } & Voltage & $220 \mathrm{~V}-50 \mathrm{~Hz}$ \\
\cline { 2 - 3 } & Body & Aluminium injection \\
\cline { 2 - 3 } & Glass & Impact resistance tempered glass. \\
\cline { 2 - 3 } & Reflector & High-efficiency pure aluminium \\
\cline { 2 - 3 } & Weight & 13.000 gr \\
\hline
\end{tabular}

\subsection{The Length of the Threshold Zone}

The first step in the design is to know the target length of the threshold zone. The length can be calculated by using the formula as defined in equation (1):

$$
D_{T h}=1.154 S D-\frac{(h-1.5)}{\tan 10^{\circ}},
$$

where $D_{T h}(\mathrm{~m})$ and $S D(\mathrm{~m})$ are the threshold length and stopping distance, respectively; $h$ is the overhead clearance $(\mathrm{m})$.

Table 3 shows the relationship between the stopping distance, longitudinal slope, and longitudinal speed.

\subsection{The average Luminance of the Threshold Zone}

The average luminance of the threshold zone $L_{\mathrm{Th}}$ is related to the luminance of the surrounding outside the tunnel $L_{20}$ as defined in [9] by the following formula:

$$
L_{T h}=k L_{20},
$$

where $k$ is the ratio of the interior of the threshold zone luminance and the exterior luminance of the tunnel; the $k$ factor is also related to the longitudinal speed and the traffic volume. The $k$ factor values are given in Table 4 for various longitudinal speed and traffic volume.

After computing, the threshold zone length and average luminance are $30 \mathrm{~m}$ and $83.47 \mathrm{~cd} / \mathrm{m}^{2}$. According to the criteria, the number of luminaires is determined. The $\mathrm{x}$-axis, $\mathrm{y}$-axis position of the luminaires determines an optimum value of average luminance, overall uniformity $U 0$, and longitudinal uniformity $U l$. The interval
Table 2. Data Used in the Design

\begin{tabular}{|c|c|}
\hline Longitudinal slope & $2 \%$ \\
\hline Stopping distance, $S D$ & $54 \mathrm{~m}$ \\
\hline Overhead clearance $h$ & $7.2 \mathrm{~m}$ \\
\hline Longitudinal speed of the tunnel & $60 \mathrm{~km} / \mathrm{h}$ \\
\hline
\end{tabular}

between the luminaires is estimated to $2 \mathrm{~m}$ spacing, and hence 16 luminaires are used in the design. The design of the tunnel is done by respecting these parameters in LITSTAR4D tunnel lighting software.

\section{DESIGN OF THE HCL-TN LED LUMINAIRE USING LITSTAR4D}

LITSTAR4D is an excellent lighting design software with many features that permit interior lighting design for buildings, road lighting, and tunnel lighting[10]. It is a user-friendly software owned by an Italian company OxyTech. The design in this software can be done by using either Adrian's picture of the tunnel or providing the value surrounding luminance in case of a fixed value of threshold zone luminance. The latter method is used in this study as our threshold zone luminance is fixed. Fig. 4 shows the designed by use of software threshold zone. The road is two-lane one way, and the luminaires are placed on each side of the lane with an interval space distance of $2 \mathrm{~m}$ from each other. The distance between the two opposite luminaires on the $y$-axis is $6 \mathrm{~m}$.

\section{RESULTS AND DISCUSSION}

This study aims to achieve the required average luminance for the threshold zone of the Huashuyan tunnel using the parameters in [2] by installing HCL-TN 


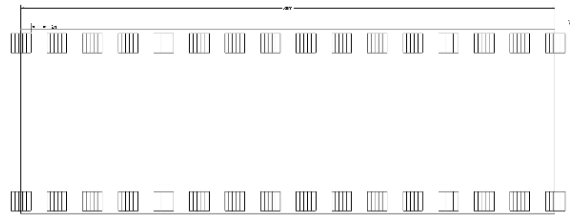

a)

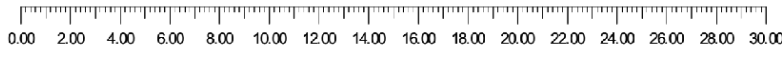
c)

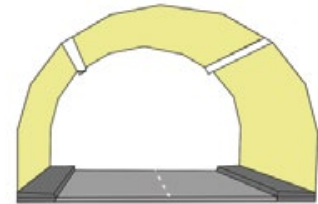

b)

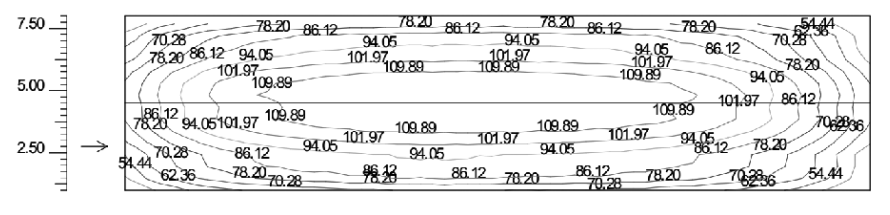

LED LITPA luminaires by simulating in LITSTAR4D software [8]. After imputing the appropriate parameters into the software, a lighting calculation was performed at the height of $1.5 \mathrm{~m}$ and over a $30 \mathrm{~m}$ length of the threshold zone. The simulation results obtained are tabulated in Table 5. The average luminance is 91.15 $\mathrm{cd} / \mathrm{m}^{2}$, which is higher than the required average luminance of $83.47 \mathrm{~cd} / \mathrm{m}^{2}$ of the threshold zone in the tunnel but less than the average luminance of the solar optical fibre with a value of $181.31 \mathrm{~cd} / \mathrm{m}^{2}$. Therefore, we can say that the luminaire met the requirement. The overall uniformity $\mathrm{U}_{0}$ of 0.42 is above the standard limit as prescribed in [9]. The longitudinal uniformity $\mathrm{U}_{\mathrm{L}}$ is 0.45 and did not fulfil the required value of 0.6 or higher, as seen in Table 5, and the relative threshold increased the value by $1.7 \%$ and met the standard. Fig. 5 shows the isoluminance distribution of the LED luminaire used in the simulation of the threshold zone of the Huashuyan tunnel.

Comparatively, the average luminance result obtained in this simulation yielded a good outcome, though the solar optical fibre lighting gave a better result for the average luminance of $181.31 \mathrm{~cd} / \mathrm{m}^{2}$ compared to that of HCL-TN LED luminaires with an average luminance of $91.15 \mathrm{~cd} / \mathrm{m}^{2}$.

\section{CONCLUSION}

To solve the problem of global warming and excessive energy consumption, scientists strive to find a suitable method to solve these issues. One of their discoveries is the usage of LED light, which is environmentally friendly and a source of energy-saving. This type of light source has excellent photometric properties, hence a good solution. In many countries, this luminaire is often used in tunnels and buildings. In this paper, we used LED light for the illumination of the threshold zone of
Huashuyan Tunnel. The length of the threshold zone was calculated using equation (1) above to determine the areas luminance requirement. The length was estimated to be $30 \mathrm{~m}$, considering the longitudinal slope, stopping distance, and the overhead clearance of the Huashuyan Tunnel. The threshold zone luminance was computed using equation (2). The luminance is related to the length of the tunnel $L_{\mathrm{Th}}$ and the luminance of the surrounding outside the tunnel $L_{20}$, which was estimated to $83.47 \mathrm{~cd} / \mathrm{m}^{2}$. The number of the LED luminaires was determined by considering all necessary parameters such as the width and height of the tunnel. The threshold zone was simulated by the LISTAR4D software using HCL-TN LITPA luminaires. The average luminance was found to be $91.15 \mathrm{~cd} / \mathrm{m}^{2}$, far beyond the required average luminance of $83.47 \mathrm{~cd} / \mathrm{m}^{2}$ of the tunnel. Luminance uniformity, threshold increases were $0.42 \%$ and $1.7 \%$ as in frame of CIE88:2004 international standard. Hence, the luminaires used in this simulation well met the design characteristics of the tunnel.

The comparative study was done for the illumination of the threshold zone of the Huashuyan tunnel. In this work, a simulation of the threshold zone has been done in the LITSTAR4D software. The calculation in the software was done according to the requirement of the threshold zone as given in [2]. The result of the simulation is then compared with the previous work, done in the literature. Even though the LED luminaires met

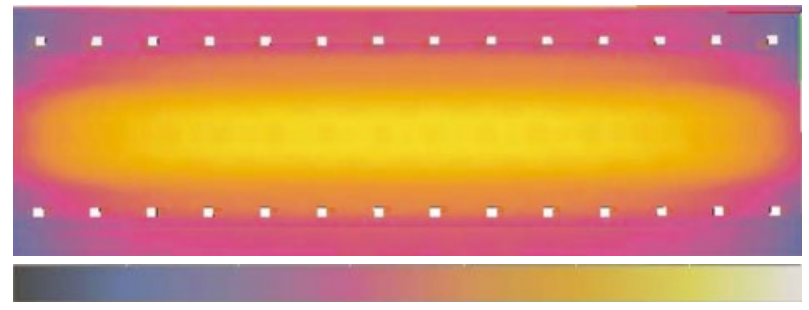

Fig. 5. The 3D view of the simulation illuminance 
Table 3. The Stopping Sight Distance SD for Highway Tunnel Lighting (m)

\begin{tabular}{|c|c|c|c|c|c|c|c|c|c|}
\hline Design speed (km/h) & \multicolumn{9}{|c|}{ Longitudinal slope (o) } \\
\hline & -4 & -3 & -2 & -1 & 0 & 1 & 2 & 3 & 4 \\
\hline 100 & 179 & 173 & 168 & 163 & 158 & 154 & 149 & 145 & 142 \\
\hline 80 & 112 & 110 & 106 & 103 & 100 & 98 & 95 & 93 & 90 \\
\hline 60 & 62 & 60 & 58 & 57 & 56 & 55 & 54 & 53 & 52 \\
\hline 40 & 29 & 28 & 27 & 27 & 26 & 26 & 25 & 25 & 25 \\
\hline
\end{tabular}

Table 4. The Relationship Between Reduction Coefficient, Design Speed and Traffic Volume of the Luminance in the Threshold Zone

\begin{tabular}{|c|c|c|c|c|c|}
\hline \multicolumn{7}{|c|}{ Design traffic volume $\boldsymbol{N}(\mathbf{v} / \mathbf{h}) \boldsymbol{k}$} \\
\hline \multicolumn{7}{|c|}{ Design speed $(\mathrm{km} / \mathrm{h})$} \\
\hline Two-lane two-way traffic & Two-lane one-way traffic & 100 & 80 & 60 & 40 \\
\hline$\geq 2400$ & $\geq 700$ & 0.045 & 0.035 & 0.022 & 0.012 \\
\hline$\geq 1300$ & $\geq 360$ & 0.035 & 0.025 & 0.015 & 0.015 \\
\hline
\end{tabular}

Table 5. Simulation Results and the CIE88:2004 Standard

\begin{tabular}{|c|c|c|}
\hline Photometric parameters & HCL-TN LED & CIE88:2004 standard \\
\hline Average illuminance, $E_{\mathrm{AV}}(\mathrm{lx})$ & 1236.55 & - \\
\hline Average luminance, $L_{\mathrm{AV}}\left(\mathrm{cd} / \mathrm{m}^{2}\right)$ & 91.15 & - \\
\hline Overall uniformity, $U_{0}$ & 0.42 & $\geq 0.4$ \\
\hline Longitudinal uniformity, $U_{L}$ & 0.45 & $\leq 0.6$ \\
\hline Threshold increase, $\mathrm{TI}, \%$ & 1.7 & $\leq 15$ \\
\hline
\end{tabular}

the requirements, the solar fibre optic still performed better with a higher value of the average luminance of $181.31 \mathrm{~cd} / \mathrm{m}^{2}$ compared to the $91.15 \mathrm{~cd} / \mathrm{m}^{2}$ average luminance of the LED fixtures.

The above findings were then compared with the existing work; it was noticed despite the LED luminaires well met the design requirement of the tunnel, the solar optical fibre system performance is higher with average luminance of $181.31 \mathrm{~cd} / \mathrm{m}^{2}$ compared to $91.15 \mathrm{~cd} / \mathrm{m}^{2}$ for the LED luminaires.

In conclusion, though the HCL-TN LED did not surpass the solar optical fibre system, it is suitable to be used in tunnel illumination.

Although all the LED lights used in this work are energy efficient and cheap they can be switch on (ignited) only by using electrical lighting systems, that is, grid-dependent systems. However, in case the tunnel in which they are used is a remote area, or in the event of a natural disaster such as storms and earthquake, it will be obliged for us to use an Off-grid system using solar energy. In contrast, a solar optical fibre system does not need any source of energy. It depends only on the availability of daylight. Therefore, the LED lighting system is more advantageous as it can be used both for daylighting and at night.

\section{ACKNOWLEDGEMENT}

The authors thank LITPA Lighting and the OxyTech Companies for their support to conduct this research work.

\section{REFERENCES}

1. Drakou D., Burattini C., Bisegna F., and Gugliermetti F. Study of a daylight 'filter' zone in tunnels // 2015 IEEE15th Int. Conf. Environ. Electr. Eng. EEEIC2015 - Conf. Proc., 2015, pp. 649-652, DOI: 10.1109/EEEIC.2015.7165241.

2. Qin X., Zhang X., Qi S., and Han H. Design of solar optical fibre lighting system for enhanced lighting in highway tunnel threshold zone: A case study of huashuyan tunnel in China // Int. J. Photoenergy, Vol. 2015, 2015, DOI: $10.1155 / 2015 / 471364$.

3. B. Liang et al. Lighting for road tunnels: The influence of CCT of light sources on reaction 
time // Displays, 2020, Vol. 61, p. 101931, DOI: 10.1016/j. displa.2019.101931.

4 Solar Lighting AB Paper, Parans W. The Importance of Illumination and Natural Lighting for Tunnels // p. 18, 2020, Available Online: https://www.parans.com/.

5. Sataloff R.T., Johns M.M., and Kost K.M. Solar lighting system Himawari // p. 5, 2019, Available Online: https://www.himawari-net.co.jp.

6. Deepak P.R. and Madhu Sharma. Fiber Optic Daylighting System // Int. J. Eng. Res., 2015, Vol. 4, \#03, pp. 637-639, DOI: $10.17577 /$ ijertv4is030738.

7. Gil-Martín L. M., Gómez-Guzmán A., and PeñaGarcía A. Use of diffusers materials to improve the ho- mogeneity of sunlight under pergolas installed in road tunnels portals for energy savings // Tunn. Undergr. Sp. Technol., 2015, Vol. 48, pp. 123-128, DOI: 10.1016/j. tust.2015.03.001.

8. Lightiing L. HCL-TN Litpa Tunnel Luminaire / Catalogue, p. 2, Available Online: https://litpa.com/indoor $/$ ? GucID=37\&SCatID=129\&\&gl=37.

9. International Commission on Illumination, "Guide for the lighting of road tunnels and underpasses," p. 40, 2004.

10. T.P. User, "LITESTAR10 Tunnel Plus User 's Manual," no. June 2011.

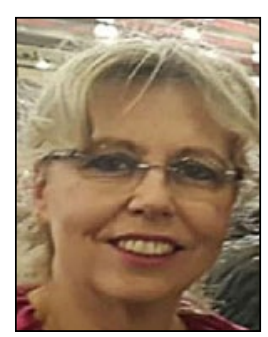

\section{Canan Perdahci,}

Ph. D., Associate Professor. She received the B. E. and M. Sc. degrees in electrical engineering from the University of Yildiz, Istanbul, Turkey, in 1991 and 1993, respectively, and the $\mathrm{Ph}$. D. degree in electrical engineering from the University of Kocaeli, Kocaeli, Turkey, in 1997.

In 1992, she joined the Department of Electrical Engineering, University of Kocaeli, as a Lecturer, and in 2019 became an Associate Professor. Her current research interests include LED lighting, UVC, power quality, tunnel lighting, road lighting

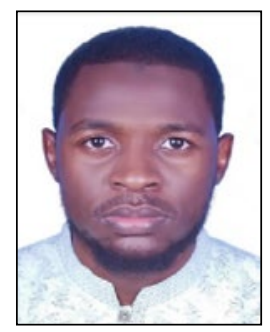

\section{Yahya Sogodok}

received a B. Sc. degree in Electrical and Electronics from the Islamic University of Technology, Dhaka, Bangladesh, in 2018. Currently, he is a master student in the Department of Electrical Engineering at the University of Kocaeli. His current research interests include LED lighting, UVC, tunnel lighting, road lighting 